\title{
Notas sobre la práctica de fútbol en un barrio indígena periurbano. Observaciones para la generación de políticas públicas
}

Ceszli, Federico

UBA, Argentina

UAM-Iztapalapa, México

IDAES-UNSAM, Argentina

Instituto Nacional de Asuntos Indígenas, Argentina

Cita sugerida: Ceszli, F.(2018). Notas sobre la práctica de fútbol en un barrio indígena periurbano. Observaciones para la generación de políticas públicas. Cuestiones de Sociología, 18, e054. https://doi.org/10.24215/23468904e054 


\section{Notas sobre la práctica de fútbol en un barrio indígena periurbano. Observaciones para la generación de políticas públicas}

Notes on soccer practice in a peri-urban indigenous neighborhood. Observations for the generation of public policies.

Federico Ceszli

$U B A$, Argentina

UAM-Iztapalapa, México

IDAES-UNSAM, Argentina

Instituto Nacional de Asuntos Indigenas, Argentina

\section{Resumen:}

El artículo presenta los resultados iniciales de una etnografía realizada en un barrio indígena periurbano con los jóvenes que se reúnen los sábados a jugar al fútbol. El método fue centralmente la observación participante y el objetivo consistió en observar el rol que ocupa el fútbol en dicho contexto social para ofrecer elementos que permitan delinear políticas públicas.

Entre los resultados salientes se incluye la desmitificación de que la práctica de fútbol en sí posea un rol preventivo para la prevención del consumo de drogas (como suele mencionarse en comunidades indígenas), la comprensión de las lógicas barriales de práctica de dicho deporte y el potencial espacio para la aplicación de tres metodologías para el desarrollo comunitario a partir del fútbol.

PaLabras ClaVe: Fútbol, indígena, política pública, drogas.

\section{Abstract:}

The article presents the initial results of an ethnography carried out in a peri-urban indigenous neighborhood with young people who get together on Saturdays to play football. The method was centrally participant observation and the objective was to observe the role played by football in this social context to offer elements that allow the development of public policies.

Among the outgoing results is the demystification that the practice of soccer itself has a preventive role for the prevention of drug use (as is often mentioned in indigenous communities), the understanding of the neighborhood logics of practice and the potential space for the application of three methodologies for community development from football.

KEYWORDS: soccer, indigenous, public policies, drugs.

En el presente trabajo me propongo exponer algunos resultados de la etnografía realizada en un barrio indígena situado en la zona norte del conurbano bonaerense. El estudio consistió en 15 visitas que se llevaron a cabo entre mayo y diciembre de 2017, en todos los casos para observar y participar de las actividades que realizan los jóvenes que se reúnen a jugar al fútbol en la cancha de fútbol del barrio. El objetivo de la etnografía consistió en indagar el rol que cumple la práctica de fútbol en la comunidad, observar las representaciones a las que está asociado entre los jóvenes y las relaciones que ellos establecen a partir de jugarlo, con el objetivo de recopilar información para pensar políticas de desarrollo comunitario a través del deporte.

Antes de iniciar el relato es preciso hacer una breve referencia al modo en que arribé a la comunidad, ya que es un elemento que explica parte de las motivaciones del estudio y que permite comprender por qué se realizó la etnografía únicamente los sábados. De lunes a viernes me desempeño como técnico profesional en el Instituto Nacional de Asuntos Indígenas (INAI), organismo estatal dedicado al desarrollo y ejecución de políticas públicas indígenas. Una parte de las acciones del INAI se realiza a través de las demandas que realizan las comunidades. A lo largo de 2016 habían llegado 13 notas con solicitudes de materiales deportivos, infraestructura o profesores de educación física, con una recurrencia particular: en varias de ellas se hacía 
referencia a que el deporte constituye una respuesta para prevenir o resolver consumos problemáticos de alcohol y drogas en los jóvenes .

De modo que el presente trabajo tuvo entre sus motivaciones la necesidad de comenzar a recopilar información sobre la relación que existe en una comunidad específica con el deporte y en particular con el fútbol, deporte que es además el mencionado con mayor recurrencia. En las presentes notas abordaremos, en consecuencia, la influencia del fútbol en la producción de identidades juveniles, la mediación del dinero en los partidos y, brevemente, algunas formas de organización imaginada por los jóvenes en torno de la Comisión de Deportes de la comunidad.

En términos generales, el método que se puso en práctica fue la observación participante. A eso se agregaron dos entrevistas -una colectiva y una individual-, la indagación de publicaciones en redes sociales y finalmente el haberme sumado a los partidos de fútbol, algo que bien podría ser incorporado en la observación participante. No se utilizaron otros métodos porque se priorizó a lo largo del proceso la construcción de un vínculo de confianza con los jóvenes, e incluso la entrevista individual surgió como resultado de que el propio entrevistado la propuso.

La cancha de fútbol mide estimativamente unos 70 metros de largo por 30 y cuenta con arcos de hierro algo más pequeños que los profesionales. No tiene techo ni partes cubiertas, es de tierra, tiene pozos, algunas piedras y un desnivel importante que va de punta a punta sobre uno de los laterales. Se trata del campo de juego más grande de la zona -todos los demás son para 5 jugadores por equipo- y si bien allí 8 contra 8 pueden jugar cómodos, los chicos cuentan que en el pasado albergaba a 11 por lado y que fue siendo reducida porque las casas y calles internas fueron ocupando cada vez más lugar. Por este motivo la cancha es, además, un espacio en disputa, ya que se trata de una porción de tierra comunitaria donde se podrían ubicar las casas de los jóvenes que a medida que crecen se independizan y forman nuevas familias, o las de parientesprovenientes de sus familias de origenque quieran sumarse al barrio.

El grupo con el que se trabajó está compuesto por un promedio de 25 varones de entre 18 y 28 años que se identifican como pertenecientes a la etnia Qom/Toba y que pertenecen de hecho a una comunidad inscripta ante el Estado. Algunos de ellos nacieron en Buenos Aires y otros en Chaco y Formosa, y poseen entre sus puntos de encuentro semanal el partido de fútbol de los sábados a la tarde. Aproximadamente un quinto de los jóvenes cuenta con trabajo estable, dos asisten al colegio secundario y no trabajan, y un tercero está terminando la secundaria en un bachillerato para adultos. Alguno que otro, además, parece recibir ingresos mediante el comercio menor de estupefacientes, y muchos de ellos acceden a planes sociales y de trabajo a través de una cooperativa, que como contraprestación solicita que se hagan tareas de mantenimiento e infraestructura en el propio barrio o en otros. Sólo uno estudia una carrera terciaria -el profesorado de Educación Física- y un segundo me comentó que le gustaría estudiar artes visuales, pero que en la Universidad Pública más cercana no había cupo para los estudiantes que no vivieran allí, de modo que iba a intentar ingresar en Educación Física, donde el acceso es más sencillo. Dos hermanos son letristas y cantantes de hip hop,y uno de los jóvenes forma parte de las divisiones inferiores de un equipo de la tercera división del fútbol argentino y vive en la pensión de dicho club, motivo por el cual no lo pude conocer en persona.

\section{Cómo SE JUEGa AL FÚtboL}

Los sábados los jóvenes se reúnen entre las 15:30 y las 18hs, cuando comienzan a juntarse en el borde de la cancha de fútbol, exactamente debajo de un alero donde pueden ser vistos desde la calle central del barrio por el resto de los jóvenes y que es percibido por ellos como su "parada", su espacio de pertenencia como grupo.

A veces aparecen con música en el celular o con algún parlante con conexión bluetooth, uno trae una pelotay se empieza a "pelotear": uno va al arco y el resto dispara desde media distancia,o se arma un juego informal de cabezazos hasta que se reúne alos 14 o 16 jugadores provistos de dinero que son necesarios para empezar un partido. En general todos tienen y se acomodan en la cancha para jugar, pero antes de empezar 
el partido alguno pregunta de manera abierta por cuánto van a jugar, y un referente de cada equipo los va mirando a todos para confirmar que tienen el dinero. Si no se llega al número pueden armar un campeonato de penales individuales o en parejas -uno patea y el otro ataja, en equipos de dos- o ir a la cancha de Villa Francisco a jugar contra el barrio vecino. No juegan todos: aquellos cuyo consumo de alcohol o drogas parece problemático no se suman, y los que no tienen dinero se quedan mirando el partido desde afuera de la cancha

Siempre se juega con dinero de por medio. Cada jugador pone 5, 10 o 20 pesos y el equipo ganador se lleva y distribuye el dinero del perdedor. Si un jugador no tiene, le pide a otro si puede poner su parte, pero completar el dinero del equipo es central en la dinámica de juego. Cuanto más hay en juego, mayor es el nivel de competitividad y, en consecuencia, mayor sentido tiene jugarlo, de modo que para ellos un partido sin dinero sería puramente lúdico, casi en tono de broma, desprovisto de intensidad.

Pese a la importancia que posee la apuesta de dinero de cara al partido, pueden darse situaciones en que equipos derrotados no entreguen su dinero al finalizar el partido: no siempre el partido lo gana con claridad un equipo -cuando alguien hace un gol dudoso en el último minuto y con ese gol su equipo gana-, o si el perdedor siente que a lo largo del partido se vio perjudicado y no está dispuesto a asumir la derrota. Estas situaciones se ven incrementadas sobre todo cuando el partido se juega con un nivel de competitividad mayor, como en los encuentros contra Villa Francisco, el barrio vecino con quienes los jóvenes del barrio tienen su mayor rivalidad. Cuando el partido es entre los propios jóvenes de la comunidad, en general el dinero ganado se utiliza para comprar bebidas que luego consumen por igual los integrantes de ambos equipos y los que no jugaron. Esto no es así cuando se juega contra otro barrio, y mi impresión es que dos de los sentidos que subyacen a esta práctica es que el fútbol permite beber o disfrutar a costa del otro, del perdedor, y que la bebida fue provista por el esfuerzo realizado en el fútbol.

Asimismo, observo que la presencia del dinero en cada partido es una práctica que reproduce en menor escala la experiencia de los torneos. En mis visitas escuché que los jóvenes del barrio participan de dos tipos de certámenes: los locales, que juegan esporádicamente y que consisten en competir a lo largo de un sábado contra un número mayor de equipos (lo que incrementa la suma de dinero que pone cada equipo y en consecuencia el botín para el ganador) y el torneo anual que se juega en la provincia de Chaco todos los $1^{\circ} \mathrm{de}$ mayo, donde en paralelo a un encuentro religioso se realiza un torneo de fútbol en el que juegan numerosos equipos y donde el campeón se lleva el monto total. Sobre ese torneo, uno de los chicos mencionó que su padre había competido y que había "mil equipos, entonces imaginate toda la plata por la que se jugaba".

Jugar al fútbol también posee una dimensión identitaria y de género (masculino), que aparece con mayor preponderancia cuando los jóvenes juegan contra "los paraguayos" (los varones de una comunidad paraguaya vecina) y especialmente contra el equipo de Villa Francisco. Con los jóvenes de este barrio la relación es tensa y los partidos de fútbol, más competitivos, lo que para ellos los hace "más divertidos". Pero no siempre juegan contra Villa Francisco, ni todos juegan: dicen que es recurrente que los partidos terminen en golpes de puño. Esta rivalidad surge de la cercanía geográfica y en consecuencia de cierta relación de alteridad que los constituye como grupo, pero también de peleas por problemas de droga, de peleas en fiestas de 15 barriales, de amigos que se defienden cuando uno es atacado y de revanchas si hubiera habido una pelea desigual en número.

En el recuerdo de los jóvenes también está la idea de que en el pasadolos varones de Villa Francisco "se abusaban" de sus propios padres, quienes por evitar las peleas concedían las derrotas incluso en la cancha del barrio indígena. Por eso una vez uno de los chicos me comentó que "nosotros somos la generación que hizo respetar al barrio", es decir que para hacer respetar fue preciso no dejarse avasallar y pelearse para defenderse de las agresiones ajenas si hubiera sido necesario.

En estos casos observo que el fútbol se torna con más claridad un espacio de expresión de masculinidades en términos de "aguante". Pero a diferencia de lo que observé en trabajos previos sobre grupos de hinchadas de fútbol (Czesli 2014), no percibí que entre los chicos del barrio Qom/Toba hubiera una clasificación entre "machos" y "putos" a partir del combate físico - aunque sí numerosos chistes a partir de aludir a un individuo 
como homosexual. De hecho, a lo largo de la etnografía las peleas y los relatos sobre estas estuvieron apenas presentes. Sin embargo, la necesidad de imponerse con el cuerpo en el barrio o en el partido (que por ser más competitivos implican jugar con más fuerza) es similar y está ligada a "hacerse respetar", a cuidar la propia cancha o la propia parada, elementos en torno de los cuales se construye la masculinidad. La práctica de fútbol y específicamente la alteridad con Villa Francisco aporta entonces elementos que contribuyen a la constitución identitaria de los jóvenes como varones, como grupo identificado como indígena y como "generación" a partir de distinguirse de generaciones previas de la propia comunidad, quienes en el relato no se habían hecho respetar. Si bien la identidad indígena excede y precede la llegada al barrio y el encuentro con Villa Francisco (me refiero a que no percibo que Villa Francisco opere aquí como otredad relacional a partir de la cual los chicos Qom se constituyen como indígenas), indudablemente son esos partidos uno de los espacios donde esa identidad se actualiza y donde los chicos del barrio se hacen respetar como grupo generacional.

A lo largo de toda la tarde son pocas las personas que se reúnen a mirarlos jugar, y las mujeres prácticamente nunca se hacen presentes. El fútbol y la "parada" resultan espacios masculinos, a tal punto que en una ocasión la esposa de uno de los chicos necesitaba decirle algo, y se asomó en el alero hasta que alguien la vio y le avisó al chico que lo estaban buscando. Mientras se juega, los chicos que esperan miran el partido y en ocasiones beben, fuman marihuana y también consumen cocaína, observación que me hizo dudar de que consumos y deporte en la práctica vayan por separado, como los discursos nativos expresaron, tanto en las demandas que llegaron al INAI como en testimonios que los jóvenes manifestaron en entrevistas:

- P: ¿Por qué para ustedes es tan importante que la cancha esté 10 puntos?

- E3: Es porque es importante...

- E1: Es un sueño que...

- E3: Los chicos crecen (...) los chicos crecen son chiquitos... la mejor, y antes de todo para mí, es un buen futuro para un pibe que juega, que juega fútbol, antes de agarrar una cosa, otra cosa. Uno mismo va, como padre, va y lo aconseja, ¿viste? Siendo que la cosa esté bien, lo que está haciendo, antes de agarrar y quedarse en la calle.

- P: No entiendo... ¿Cuál es la relación entre el fútbol, deporte y quedarse o no quedarse en la calle?

- E3: No, porque el fútbol también, te, te rescata de muchas cosas, viste, te rescata de muchas cosas. El fútbol es lindo, y más cuando sos chico y te gusta jugar al fútbol y mejor. (...)

- P: ¿Conocen casos de gente que a través del fútbol se haya rescatado?

- E3: Sí... muchos.

- E2: Había, había uno que, tenía como 14, 15 años que, que le mandaba meta faso, tomaba mucho (...) y el chabón iba en un club, todo, hasta que lo ganó nomás la calle y ya estaba dejando el fútbol y lo llevaron para el Chaco, y ahí lo estaban llamando del club, que jugaba bien todo, es un pibe del barrio, y vino entró en el club y decidió quedarse ahí adentro, él juega en Cañuelas ahora, él juega ahí ahora.

- P: ¿Es profesional?

- E2: No, todavía es chico, tiene 17 años, y nada, decidió quedarse ahí adentro y salir de las drogas, todo eso.

Los partidos en general se extienden hasta que se dificulta ver la pelota porque se hace de noche, y entonces los chicos vuelven a "la parada" y continúan con el espacio de encuentro. Algunos se retiran, pero son escasos los que no adoptaron dichos consumos. Y si bien tanto el espacio futbolístico como la ronda posterior parecen tener como denominador común la construcción de grupo, a lo largo de la etnografía y motivado por la oposición que aparece en la comunidad entre "deporte" y “drogas" me pregunté si en la práctica son acontecimientos inseparables(es decir, si inevitablemente en la situación social estudiada jugar al fútbol está ligado al encuentro posterior y a dichos consumos). Las observaciones realizadas me permiten sostener que la relación no es necesaria, idea que sostengo sobre todo a partir de observar que algunos jóvenes juegan y no se quedan al encuentro posterior, o se quedan pero no consumen. Un elemento que puede contribuir a refrendar esta idea surgió de la constatación que entre los adultos que asisten a las Iglesias del barrio no es criticada la práctica de fútbol ni es asociada a "estar en la calle" o al consumo de drogas. En cambio, parecen 
sostener una imagen del futbol como un deporte ligado a la salud, y como un camino para evitar que los chicos caigan en las drogas.

Jugar al fútbol también está asociado a la posibilidad de un desarrollo profesional. Cuando en una entrevista colectiva con cuatro de los jóvenes se refirieron a los objetivos que tienen como Comisión de Deportes, mencionaron tener una cancha "bien presentable, con césped, reflectores, con un medidor [de luz] (...) para los reflectores de la cancha..." y en segundo término, la idea de hacer "un club aborigen en el barrio". Tener una cancha "bien presentable" está asociado a una suerte de orgullo barrial, de honor de la comunidad ante el resto de los barrios de la zona. Incluso, como se puede ver en el siguiente extracto de la misma entrevista, los jóvenes consideran que de constituir un club aborigen "saldrían grandes jugadores de acá", una figura que podría estar asociada a dos significantes: a los mitos contemporáneos como el del futbolista-estrella que "sale de su barrio para consagrarse en el exterior pero nunca se olvida de dónde salió" y a la idea de que en el barrio hay materia prima de calidad que está siendo desaprovechada.

- P: ¿Qué se imaginan que pasaría si hubiera un club?

- E1: [afirma con fuerza] Saldrían grandes jugadores de acá.

- E3: Síii, también.

- E1: ¿Sabés los guachines cómo juegan? Son re chicos, viste, pero les re gusta y juegan re bien además, son... chicos.

- E3: Yo tengo a mi nene chiquitito, viste, 4 años, le gusta jugar al fútbol, yo lo meto acá en el barrio y va a jugar, ya lo tengo... Es, es lindo hacer un club.

- E1: Sí... más que nada para los chicos viste, para los más chicos

La idea de la materia prima desaprovechada se puede observar en la frase del entrevistado E1 “...saldrían grandes jugadores de acá (...)Además, son... chicos", ya que probablemente ese último "son chicos" remita a la idea de que aún tienen tiempo de realizar carrera de futbolista, a diferencia de los entrevistados, que con un promedio de veinte años ya perdieron su posibilidad.

Pero al mismo tiempo, en el discurso aparece siempre la figura de "hacerlo por los chicos", es decir que hablan de mejorar la cancha o hacer el club no para ellos mismos sino por esos otros que están creciendo y que podrían "caer" en malos hábitos de salud. Mi interpretación, en este caso, oscila entre la apelación demagógica para conseguir recursos (la imagen del niño se asocia a la inocencia, nadie podría oponerse al deseo de desarrollo y al juego de la infancia del barrio) frente a una figura como la mía que en última instancia pertenecía al Estado, y la idea de que si ellos como jóvenes pueden ya no tener la posibilidad de revertir su destino, "el futuro" de los niños y niñas aún está intacto. En este sentido, cuando el joven me comentó que su sueño era generar un club indígena, mencionó que su sueño había sido ser futbolista profesional, pero que no había tenido la oportunidad y en consecuencia quería que al menos los chicos del barrio sí la tuvieran.

\section{APUNTES PARA LA INTERVENCIÓN}

El trabajo etnográfico permitió observar algunos de los sentidos con los que la práctica de fútbol está asociada en el barrio indígena. Lo primero que podemos mencionar es que el fútbol ocupa un espacio de ocio central en un contexto de pocos accesos a otras formas de divertimento, recreación y producción cultural. Su práctica es además tradicional, porque existen numerosos relatos de cuando eran sus padres quienes lo jugaban, ya que está ligado a eventos religiosos y es jugado desde la infancia, de modo que es una práctica naturalizada. Asimismo, hemos visto que se juega por dinero, y que es ese el elemento que le da al partido el sentido de que no es algo únicamente lúdico, comprometiéndolos a esforzarse.

Teniendo en cuenta que uno de los objetivos del presente trabajo consiste en sugerir posibles líneas de acción a partir de la experiencia de campo presentada, dedicaré las próximas líneas a esbozar brevemente caminos potenciales de acción. En primer término, entonces, puede no ser desatinadopensar iniciativas que no sólo trabajen el fútbol desde una perspectiva lúdica o social sino que aprovechen además las aspiraciones de 
profesionalismo de forma motivacional (en línea con las observaciones de Segura Trejo, 2014, p. 6), incluso a sabiendas de que la tasa de jóvenes que alcanzan la Primera División sea muy reducida (Murzi y Czesli, 2016).

Que en los partidos de la zona se juegue sin árbitros y que raramente haya conflictos dirimidos de manera violenta puede significar que haya ahí un espacio fértil para trabajar la resolución dialogada de conflictos, por ejemplo a través del método de Fútbol de 3 Tiempos promovido por FuDe o la Street Football World ${ }^{6}$ (Segura Trejo e Islas Govea, 2016). Observamos además que los jóvenes como equipo se organizan de manera precaria, lo que abre la posibilidad de trabajar en el desarrollo colectivo a partir de la generación de consensos sobre una táctica y estrategia de equipo, por ejemplo a partir del método de Investigación Acción Participativa puesto en funcionamiento por John Alexander Cárdenas en Colombia (Cárdenas-González y Casallas Torres, 2016)

Para finalizar, considero que uno de los hallazgos más interesantes del trabajo consiste en desmitificar la idea nativa y extendida en ciertas comunidades indígenasde que donde hay deporte no hay drogas, o de que la práctica deportiva y de fútbol son, en sí, mecanismos preventivos o resolutivos de consumos problemáticos. El hecho de que los jóvenes referidos en el presente trabajo esgriman al deporte como un camino para prevenir los "consumos problemáticos" entre los chicos de la comunidad, y al mismo tiempo hagan un uso de las mismas en el contexto de su práctica futbolística regular es, al menos, un eje donde hacer foco.

Es válido entonces poner en duda los presupuestos desde los que se desarrollan las políticas públicas orientadas a la "prevención de adicciones", y apostar tanto a la mirada etnográfica para indagar el lugar que se le concede a la práctica deportiva en las comunidades destinatarias y la trama de significaciones que la atraviesa. Dentro de esos presupuestos se incluye la población destinataria y los métodos de desarrollo, ya que es el trabajo territorial el que puede permitir relativizar las concepciones desde la cual dicha demanda se construye, definir la población destinataria capaz de apropiarse del deporte como herramienta de promoción social y especificar, en función de su relación con el deporte, los métodos adecuados para su puesta en funcionamiento.

\section{Referencias bibliográficas}

Bourdieu, P. (2010). El sentido práctico. Buenos Aires: Siglo Veintiuno.

Cárdenas-Gonzalez, J. y Casallas Torres H. (2016). Del deporte colectivo al juego comunitario: fortalecimiento de las organizaciones comunitarias de jóvenes por medio de la práctica del fútbol. En Guzmán Ariza (comp.),Deporte, inclusión social y experiencias comunitarias en América Latina. Villavicencio: Editorial Unillanos.

Czesli, F. (2014).Morir por Platense. Adversidad y temporalidad como estructuras perceptivas en una hinchada de fútbol. Revista del Museo de Antropología,7.

Garriga Zucal, J. (2007).Haciendo amigos a las pinas: violencia y redes sociales de una hinchada de fútbol. Buenos Aires: Prometeo Libros.

Moreira, M. V. (2005). Trofeos de guerra y hombres de honor. EnAlabarces, P. (Comp.), Hinchadas. Buenos Aires: Prometeo.

Murzi, D. y Czesli, F. (2016).De la humildad a lo mental. Un análisis comparativo del proceso de formación de futbolistas profesionales en Argentina y Francia. Apuntes de Investigacio\#n del CECYP, 28,162-182.

Segura Trejo, F. (2014). Apuntes sobre la utilización del fútbol como recurso social: hacia una descripción comparativa internacional. Documentos de Trabajo del CIDE, 285. Ciudad de México: CIDE.

Segura Trejo, F. e Islas Govea, A. (2016). El fútbol en tres tiempos sociales. Observaciones de un festival asociativo en Cajú, Río de Janeiro, Brasil, 2014. PODIUM Sport, Leisure and Tourism Review, 5(2).

\section{Notas}

1 No se brindarán mayores precisiones sobre la localidad para proteger la confidencialidad de sus integrantes. 
2 No incorporo más precisiones sobre las notas para evitar transgredir la normativa sobre información confidencial de las comunidades y sobre las acciones de gobierno.

3 Qom y Toba son términos utilizados de manera indistinta en la comunidad.

4 Tengo indicios de que a lo largo de la semana -en función de la carga laboral o estudiantil, entre otros factores- también se reúnen en grupos más pequeños, sobre todo en horario nocturno. De modo que una de las limitaciones del presente trabajo es no contar con información sobre lo que sucede en la semana, y considero que para comprender cabalmente la práctica de fútbol de los sábados como espacio de ocio masculino es preciso pensarla en relación con lo que sucede de lunes a viernes, días tradicionalmente dedicados al trabajo o estudio.

5 Se trata de un concepto presente entre los aficionados al fútbol que fue estudiado para pensar la violencia física en contextos de espectáculo futbolístico, usualmente atribuida a las "barras bravas". En dichos grupos, el "aguante" es un bien simbólico deseado porque inviste de honor a quienes lo poseen, y está ligado a las construcciones de género masculino porque la demostración de temperamento en el combate físico permite clasificar a los "machos" (los que no se acobardan en las peleas) de los "putos" (que sí se acobardan y "corren”) (Garriga Zucal 2007; Moreira 2005, entre otros).

6 http://www.fundacionfude.org.ar/; http://www.streetfootballworld.org/ 